\title{
¿Hacia una democracia paritaria en América Latina? Estrategias fallidas y esperanzas vacias
}

\author{
Towards Parity Democracy in Latin America? Ineffective Strategies \\ and Failed Expectations
}

\section{Karolina Monika Gilas}

\section{Resumen}

La construcción de la democracia paritaria requiere de un reconocimiento de los derechos de todas las personas, sin importar su género. Ese reconocimiento, a su vez, implica la garantía de capacidad plena de ejercicio de esos derechos. La literatura y el discurso han señalado que la ruta hacia la igualdad y construcción de una democracia transita por el ámbito político-electoral, que el incremento en la representación política de las mujeres llevará, tarde o temprano, a un cambio de la situación e implementación de las políticas públicas con perspectiva de género. Muchos países, incluyendo todos los países de la región, apostaron por las cuotas de género como un mecanismo efectivo y rápido para lograr la representación descriptiva de las mujeres. Sin embargo, a más de veinticinco ańos de las acciones afirmativas, la realidad de la región, vista a través de los indicadores de desigualdad de género, desmiente esa apuesta. La democracia paritaria no se logra a través de la ruta electoral. Al menos, no exclusivamente a través de esta.

\section{Palabras clave}

Género; Igualdad de Género; Paridad; Democracia.

\section{Abstract}

The construction of parity democracy requires the recognition of rights of all people, regardless of gender. This recognition, in turn, implies the guarantee of full capacity to exercise those rights. Literature and political discourse have argued that the route to equality and parity democracy goes through politics and elections, and that the increase in political representation of women sooner or later will lead us to a real change in their situation and to implementation of public policies with a gender perspective. Many countries, including all the countries in the region, supported gender quotas as an effective and fast-track mechanism to achieve descriptive representation of women. However, after more than twenty five years of affirmative action, the reality of the region denies that idea. Parity democracy is not being achieved through the electoral route. At least, not exclusively via quotas.

\section{Keywords}

Gender; Gender Equality; Parity; Democracy. 


\section{Introducción 1}

En las últimas décadas pudimos observar un cambio significativo en la región al respecto de la participación política de las mujeres. Todos los países latinoamericanos han implementado algún tipo de acción afirmativa a favor de la participación de las mujeres, y lo han hecho con resultados más que positivos. Las cuotas resultaron ser tan efectivas que la región pasó a segundo lugar en el mundo en cuanto a la representación legislativa de las mujeres, en menos de veinte años, y por encima de la mayoría de los países europeos ( $\sin$ tomar en cuenta a la región escandinava). En 1997 el promedio regional era de 13,5\%, mientras que el ańo 2015 cerró con un promedio de $27 \%$, alcanzando el doble.

Sin embargo, el objetivo de las medidas afirmativas adoptadas no es únicamente un incremento en el número de las mujeres que ejercen los cargos de representación o de gobierno; su finalidad última es la construcción de una democracia paritaria, en la que las personas de ambos géneros gozan de la plenitud de derechos y libertades. La vinculación entre ambos es la suposición de que un aumento en la presencia e influencia de las mujeres en la política paulatinamente se traduciría en la adopción de las políticas públicas sensibles a los temas de género y, con ello, se alcance la ciudadanía plena de las mujeres.

El presente artículo pretende evaluar la construcción de la democracia paritaria en la región, analizando los efectos de la representación política de las mujeres frente a otros parámetros de igualdad de género. Es decir, intenta verificar si el aumento en la representación femenina en los países de la región se ha traducido en modificación de las políticas públicas y, en consecuencia, mayor igualdad entre hombres y mujeres en otros ámbitos de la vida social: trabajo, acceso a la educación, a los servicios de salud, a mayores recursos económicos, etc.

\section{Democracia paritaria: ¿igualdad en qué?}

La igualdad en la participación se volvió prioridad en la política internacional desde la década de 1990. Tanto la Declaración de Atenas de 1992, como la Declaración de Beijing y Plataforma para la acción de 1995, establecían como objetivo de las políticas públicas alcanzar la igualdad de participación de mujeres y hombres en la toma de decisiones. Desde entonces los países democráticos han puesto mayor énfasis en el establecimiento de medidas efectivas para fomentar la representación femenina en los distintos ámbitos de la vida pública. En algunos documentos

\footnotetext{
${ }^{1}$ Agradezco a Cecilia Aida Hernández Cruz sus valiosos comentarios y largas discusiones sobre las cuotas de género que me permitieron aterrizar y concluir el presente estudio.
} 
internacionales, así como trabajos académicos, hablan incluso de la necesidad de alcanzar una democracia paritaria.

Como muchos conceptos en las ciencias sociales y en el debate público, también la democracia paritaria puede ser definida desde diferentes perspectivas. El mismo feminismo tiene diferentes posturas al respecto, desde la perspectiva de feminismo de la igualdad y de la diferencia. Así, la visión sexuada que de la representación hace que el feminismo de la diferencia, postula la paridad en los cargos como el objetivo a perseguir. La sociedad, siempre sexuada y por tanto dividida en dos grandes partes, debe ser reflejada por una representación igualmente sexuada y dividida. El feminismo de la igualdad, en cambio, considera que la paridad, como otras medidas afirmativas, no es más que un medio necesario de implementarse en las sociedades actuales y el cual debería, en un momento determinado, llevar a una igualdad real y sustantiva entre los géneros y, por lo mismo, a una verdadera universalidad de los derechos. Aunque ambas perspectivas persiguen, como fin ulterior, una participación política de las personas tanto en el sentido de oportunidades, como de presencia, en los ámbitos de la toma de decisión, la diferencia estriba en los "números" a lograr y en el tipo de medidas que se deben adoptar para conseguirlo (GONZÁLEZ et al., 2016).

La Declaración de Atenas señalaba que se trata de la "total integración, en pie de igualdad, de las mujeres en las sociedades democráticas, utilizando para ello las estrategias multidisciplinares que sean necesarias" (UNIÓN EUROPEA, 1992). En ese contexto, la demanda de paridad tiene que ver con el asumir que la democracia puede ser real solamente si se basa en el reconocimiento de los derechos y en la inclusión de todas las personas en la vida pública. Por ello, se debe buscar una representación equilibrada entre ambos géneros, con lo que tendría que lograrse una transformación de los procesos sociales, pero no solamente políticos. Una representación política de ambos géneros en torno al 50\% tendría que ir acompañada (en un momento dado) por una auténtica distribución de las responsabilidades entre ambos sexos. En ese sentido, la democracia paritaria "Va más allá de la distinción entre vida privada y pública: reconoce que la vida privada es política" (LEUCHPRECHT, 1996, p. 126).

Por supuesto, el camino hacia la democracia paritaria va a través de las acciones afirmativas de género: 
El reconocimiento de los derechos políticos de las mujeres debe concentrarse en su participación paritaria en las decisiones del estado a través de una gran variedad de mecanismos: desde la reserva de una cuota paritaria de representación en el Parlamento, gobierno nacional, gobiernos autonómicos y municipales, hasta el establecimiento de mecanismos preceptivos y vinculantes de consulta a las mujeres en caso de decisiones que les afecten específicamente. Esto requiere un sistema de control y garantía de derechos que debería incluir una instancia jurisdiccional a la que pudieran llegar los recursos y que obligase al estado (COBO, 1999, p. 34).

Algunas de las concepciones de paridad, las cercanas al feminismo de la igualdad, ponen mayor énfasis no en la participación numéricamente equitativa de mujeres y hombres en los cuerpos legislativos y demás ámbitos de representación y toma de decisiones, sino en un aspecto cualitativo que implica la presencia más allá del sentido físico, y también en el sentido simbólico y de intereses. Desde esa perspectiva, se busca una repartición equilibrada de poder entre mujeres y hombres, lo que no tiene que llevar a una igualdad numérica (AGACINSKI, 1998; ZÚÑIGA, 2005, p. 92).

Como podemos ver, la idea de la paridad no necesariamente trata de una división en dos mitades de todos los espacios públicos, más bien se busca una mejor representación que refleje en mayor medida la pluralidad de las experiencias, visiones y preferencias de la sociedad:

[...] no es una cuestión de que las mujeres representen a las mujeres, sino de dar a las mujeres tantas posibilidades de influir en el destino común como a los hombres, de permitir que las mujeres piensen en el futuro global de la sociedad y no sólo en el problema de los cuidados diarios; de lograr que la sociedad se reconozca en ellas como lo hace con sus contrapartes masculinas (DONANRD, 1994 apud SCOTT, 2012, p. 99-100).

Lograr esa meta, la de presencia e influencia, de reconocimiento de la igualdad de las mujeres, va más allá del fomento de la participación política femenina. Debe incluir la implementación de las políticas públicas que garanticen la igualdad de acceso y de ejercicio real de otros derechos fuera del plano político-electoral: en los planos económico, educativo y social. En otras palabras, una representación política de las mujeres debería estar acompańada por un cambio sustantivo en los arreglos económicos y sociales, de tal manera que se logre una igualdad plena. 


\section{Las cuotas de género: una via rápida hacia la igualdad}

Las cuotas de género son una medida afirmativa, empleada ampliamente desde la década de 1970, para contrarrestar los efectos que la discriminación y exclusión de las mujeres tiene sobre sus posibilidades de desarrollo profesional y personal (MEDINA TORRES, 2011, p. 9). Las cuotas son un mecanismo legal que implica reservar para los representantes de un grupo particular un determinado número de candidaturas, escaños, puestos, etc. En el ámbito de la participación política,

[...] buscan elevar el porcentaje de mujeres en el Parlamento o alcanzar el equilibrio de género y establecen una participación mínima de candidatas en las elecciones, por lo menos en las listas de los partidos. Adicionalmente pueden también contener disposiciones que toquen el posicionamiento de candidatas en las listas (KRENNERICH, 2009, p. 189).

La implementación de las cuotas y otras medidas afirmativas ha sido intensamente discutida. Con frecuencia, se señala que, además de ser discriminatorias hacia los hombres, son antidemocráticas, generan fraccionalización de la política y van en contra de los principios de autoorganización de los partidos políticos y de la meritocracia (IDEA, US y IPU, 2015; DAHLERUP y FREIDENVALL, 2008).

En la actualidad, está muy extendido el uso de las medidas a favor de la participación política de las mujeres, que deriva justamente del reconocimiento de sus derechos políticos y de la aceptación de la necesidad de garantizar su ejercicio efectivo. Queda claro que, para lograr ese objetivo, se necesita más que el mero reconocimiento legal de la igualdad de todas las personas. En palabras de Fernando Rey:

La experiencia histórica confirma, una y otra vez, que la identidad jurídica de trato entre mujeres y hombres, entre payos y gitanos, etc. actúa más bien como un instrumento de conservación del statu quo, más que como un punto de partida para un desarrollo futuro más igualitario. Cuando un Derecho neutral se enfrenta a un estado de desequilibrio social entre sexos, etnias, etc. y, paralelamente, se enfrenta a una situación de superior importancia del grupo de los varones, blancos y propietarios en el ámbito de las elites políticas y sociales, entonces no puede desempeñar una función de igualación y se llega, por el contrario, a una toma de partido unilateral en favor de los grupos dominantes y en detrimento de las minorías. En otras palabras, en una situación de 
desigualdad real y efectiva de las mujeres, de los gitanos, etc., la adopción de un Derecho "neutro" no es una decisión neutral. Las acciones positivas para la igualdad de oportunidades de las mujeres, los gitanos, los discapacitados, etc., no sólo tienen el aire de familia del Estado Social y su general postulado de la egalité des chances (que afecta a diversos grupos sociales en desventaja: parados, emigrantes, inmigrantes, jóvenes, etc.), sino que, como ya se ha indicado, son medidas especialmente exigidas por el constituyente. A diferencia de las políticas de apoyo mencionadas en favor de los otros grupos sociales (que también encuentran cobertura constitucional, concretamente, en el Capítulo Tercero del Título I), las acciones positivas para la igualdad en estos casos ni deben depender de las medios financieros existentes, ni deben estar condicionadas por la polémica de los partidos políticos que compiten por la mayoría en el Parlamento, ya que el objetivo a alcanzar con ellas es claro y preciso (tanto si la mayoría es de un color político como si es de otro): la "igualdad perfecta" de la que hablara J.S. MILL para ambos sexos (que no haya privilegio ni poder para uno ni incapacidad alguna para el otro). La igualdad real y efectiva entre géneros, grupos étnicos, etc. está por encima del debate político. Debe lograrse en todo caso. Se trata de un derecho fundamental, de una decisión del constituyente sustraída de la mayoría política cambiante (REY MARTÍNEZ, 2013, p. 19-20).

Los partidarios de las cuotas de género consideran que "se requiere un tratamiento diferenciado para compensar las discriminaciones existentes que generan estas desigualdades y así lograr una igualdad de hecho y una profundización de la democracia" (MOREIRA y JOHNSON, 2003, p. 17). El bajo nivel de participación de las mujeres en la vida pública y la arena política justifica la implementación de las acciones afirmativas. De acuerdo con Moreira y Johnson, las cuotas de género se justifican como una medida temporal y correctiva para hacer efectivo el derecho político de las mujeres a ser elegidas: "un derecho que se ha visto coartado en los hechos por la incidencia de relaciones históricas de subordinación y discriminación en las posibilidades reales que tengan de competir en condiciones de igualdad con los hombres" (MOREIRA y JOHNSON, 2003, p. 17). Aunque este argumento reconoce a las mujeres como un grupo social particular, "apela más al derecho de ser representantes de individuos que comparten la condición de pertenecer a un grupo social e históricamente subordinada, que a una representación de grupo, basada en una concepción de una coincidencia de intereses o necesidades entre las integrantes del mismo" (MOREIRA y JOHNSON, 2003, p. 17-18). Cerva y Ansolabehere sostienen que: 


\begin{abstract}
[...] no sería suficiente otorgar los mismos derechos a las mujeres, sino más bien trabajar en la modificación de las instituciones jurídicas y del mismo derecho, en los principios y las doctrinas jurídicas y en la toma de conciencia del ejercicio de un tipo de dominación de género por parte de los operadores del derecho. En otras palabras, desde esta mirada, se requieren cambios institucionales sostenidos por transformaciones culturales" (CERVA y ANSOLABEHERE, 2009, p. 18).
\end{abstract}

La presión ejercida por las organizaciones internacionales y feministas, así como a causa del lento incremento "natural" de la participación femenina, explican la necesidad de introducir mecanismos para incentivar la participación y representación de las mujeres y llegar a la igualdad sustancial. Así, las cuotas se han convertido en un mecanismo fundamental para lograr la igualdad de representación entre mujeres y hombres, o por lo menos de igualdad de oportunidades de presentarse al escrutinio de los votantes (DAHLERUP y FREIDENVALL, 2005, p. 29-30). Además, Aparicio y Langston añaden que "son los partidos políticos, y no los votantes, quienes controlan de manera más inmediata el acceso a las candidaturas. En este sentido, las cuotas de género no son muy distintas a otras restricciones comúnmente aceptadas en el diseño constitucional de las democracias" (APARICIO y LANGSTON, 2009, p. 7).

Sin embargo, el argumento más fuerte a favor de la cuota es el de su efectividad: la mayoría de los países donde se ha implementado, la representación legislativa de las mujeres superó el 30\% (NORRIS, 2006). Además, las cuotas permiten lograr ese aumento en poco tiempo: Dinamarca, uno de los países modelo para la equidad de género, consiguió incrementar la presencia de las mujeres en su parlamento a un 38\% en 2001, es decir, le tomó 20 años y ocho procesos electorales. En cambio, la implementación de la cuota en Costa Rica elevó la representación femenina de 19\% al 35\% en solo una elección legislativa de 2002 (DAHLERUP y FREIDENVALL, 2005, p. 28).

Existen diferentes tipos de cuota, aunque es importante señalar dos variables principales que nos permiten distinguir entre ellos. La primera variable es la relativa a dónde se introducen las cuotas: en la legislación (constitución o código electoral) o en los estatutos de los partidos. La segunda variable se refiere a dónde se pretende cambiar la proporción entre géneros: a) entre los precandidatos; b) entre los candidatos; y c) entre las personas electas (DAHLERUP, 2006, p. 19). Básicamente, hay tres modelos utilizados por los distintos países: 
- Escaños reservados: bajo este esquema, un determinado número de escaños se excluye de la competencia general y se reserva para una competencia únicamente entre candidatos mujeres (Jordania, Ruanda y Uganda);

- Cuotas legales (constitucionales y/o legislativas): establecen un porcentaje mínimo de lugares en las listas de candidatos para el género subrepresentado. Algunos países las incluyen a nivel constitucional (México, Nepal, Filipinas y Uganda) o en la legislación electoral (Bélgica, Eslovenia y Francia);

- Cuotas de partidos: en algunos casos, los partidos políticos incluyen las cuotas en sus normas internas, aun cuando no existe una obligación legal para ello (Alemania, Noruega y Suecia).

En América Latina, la implementación de las cuotas de género data desde 1991, cuando en Argentina, mediante la Ley de Cupos, se estableció una cuota del 30 por ciento de candidaturas de representación para las mujeres (PESCHARD, 2002, p. 174). Los demás países de la región siguieron ese ejemplo y, durante la década de los noventa, aprobaron algún tipo de cuota de género. Los diseños fueron variados $y$, por tanto, generaron diferentes resultados. El estudio de Medina Torres, que revisa la implementación de las cuotas en los dieciocho países de América Latina en sus diversos momentos históricos y de diseńo (MEDINA TORRES, 2011; PESCHARD, 2002), demuestra claramente qué factores son la clave del éxito:

- La proporción de la cuota. Como es de esperarse, ante un mayor número de escaños destinados a las mujeres, mayor probabilidad de que algunas de ellas queden electas.

- La alternancia en las listas. La alternancia refuerza la cuota al obligar a los partidos a llegar, o al menos acercarse, a la paridad en las listas.

- Aplicación de la cuota sobre lugares elegibles. Asegurar que las mujeres sean postuladas en las posiciones que tienen altas posibilidades de quedar electas fomenta la probabilidad de obtener el cargo.

- La obligatoriedad y sanciones por incumplimiento. Las cuotas voluntarias (de partido) y las cuotas legales que no están respaldadas por sanciones tienen una efectividad baja, ya que no generan incentivos correctos para modificar el comportamiento de los partidos.

Las cuotas en los países latinoamericanos tienen diferencias importantes en el diseño y, por ende, generaron diferentes resultados, como se puede observar en el siguiente gráfico: 
Grafico 1 - Evolución de la representación política de las mujeres

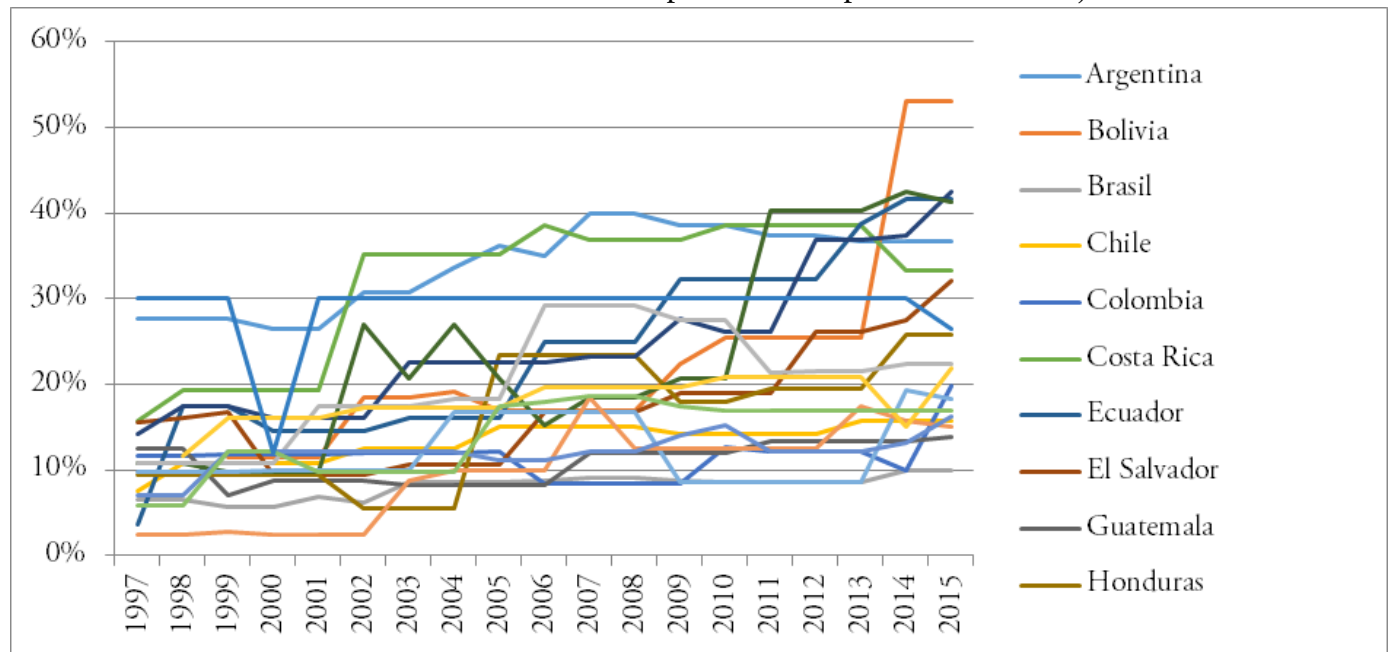

Fuente: Elaboración propia a partir de IPU (2016).

Todos los países, con la excepción de Guatemala, han registrado un incremento en la representación femenina, aunque este varía en la intensidad. El resultado final, obtenido a finales de 2015, nos permite agrupar a los países en cuatro grupos:

- Casos con efecto fuerte: Bolivia, México, Ecuador, Nicaragua, quienes lograron la representación femenina por encima del $40 \%$;

- Casos con efecto elevado; Argentina, Costa Rica y El Salvador, con resultados entre $30 \%$ y $39 \%$;

- Casos con efecto moderado: Honduras, Perú, República Dominicana, en el rango del $20 \%$ al 29\%;

- Casos con efecto débil: Colombia, Panamá, Venezuela, Uruguay, Chile, Paraguay, Guatemala, alcanzando niveles entre 10\% y 19\%;

- Caso fallido: Brasil, el único país que no logró rebasar el umbral de $10 \%$.

Debido a que la ciencia política considera que el umbral mínimo necesario de representación política de las mujeres para tener un impacto real en los procesos de toma de decisión es el 30\% (DAHLERUP, 1998), podemos considerar que todos los casos que han logrado rebasarlo son casos de éxito. Esa perspectiva nos otorga 7 casos exitosos frente a 11 casos con efecto débil. Al mismo tiempo, nos haría suponer que los casos exitosos debieron haber generado políticas públicas favorables para la igualdad de género y que, como consecuencia, deberían obtener mejores resultados en 
las mediciones respectivas. En el siguiente apartado, vamos a evaluar si se confirma esa hipótesis.

\section{El impacto de la representación femenina}

La discusión sobre las cuotas y las demás medidas necesarias para incrementar la participación de las mujeres en la vida política y, especialmente, en los cargos de dirección, tarde o temprano llega a la misma pregunta: ¿y qué diferencia hace una mayor presencia de las mujeres? Esa pregunta es especialmente relevante en el contexto de la democracia paritaria, que assume la construcción de una sociedad igualitaria, en la que el género de las personas no puede ser un factor determinante para su desempeño, participación política o desenvolvimiento en cualquier ámbito de la sociedad.

La presencia de las mujeres en los espacios de decisión suele generar expectativas de un cambio, tanto de contenido como del estilo de la política. En el fondo, muchas de esas expectativas son una expresión de estereotipos de género y se basan en la asociación de las mujeres con ciertas cualidades o características personales: se espera que sean más conciliadoras, más amables, más dispuestas a escuchar, a tomar en cuenta diferentes puntos de vista. En consecuencia, se espera que se encarguen de los "temas de mujeres": equidad de género, derechos reproductivos, niñez, educación, servicios de salud, programas sociales para los grupos desfavorecidos, entre otros. Los temas "masculinos": defensa nacional, presupuesto, seguridad pública se convierten en temas reservados para hombres. Esa división sexual de la política, en el fondo, es absurda, al asumir que esos asuntos no les interesaran o no les afectaran a las mujeres, o como si los hombres no usaran servicios de salud y no se preocuparan por la calidad de la educación que reciben sus hijos.

Al mismo tiempo, existe un número importante de investigaciones que demuestran que, efectivamente, las mujeres suelen ocuparse en mayor medida de los temas considerados como de corte social, es decir, que tienen que ver con las cuestiones de desigualdades de todo tipo y que pudiéramos considerar relacionados con el estado de bienestar. Por ejemplo, la literatura señala que las mujeres legisladoras tienden a comportarse diferente, cumpliendo con las expectativas: apoyan en mayor medida que sus contrapartes masculinos la legislación a favor de derechos reproductivos y cambio de roles de género (NORTON, 1999); apoyan programas de rehabilitación de criminales, mientras que los hombres suelen optar por castigos más severos (KATHLENE, 1995); dedican más tiempo al servicio de la comunidad que los hombres (THOMAS, 1992); con mayor frecuencia, toman en cuenta las 
opiniones de los ciudadanos y se preocupan en establecer vínculos con ellos (FOX y SCHUHMANN, 1999); su estilo de gobierno enfatiza la búsqueda de coincidencias y cooperación (TOLLESON, 1991), entre muchos otros (LAWLESS y FOX, 2012).

Algunos estudios (KANTER, 1977; DAHLERUP, 1998) sostienen que es necesario lograr una "masa crítica" de la presencia femenina, a partir de la cual su influencia en las políticas públicas sea mayor; de ahí la importancia de las medidas afirmativas en el ámbito electoral. Desde esa perspectiva, se ha señalado que el porcentaje de las mujeres en los legislativos es determinante para el contenido de la legislación aprobada y que los parlamentos con mayor presencia femenina aprueban mayor cantidad de leyes con perspectiva de género (BRATTON, 2005; THOMAS, 1991; THOMAS, 1994). En un tenor similar, algunos estudios, como el de Rodríguez, señalan también que, más allá de la agenda específica "femenina", las mujeres que llegan a dedicarse a la política suelen ser un factor favorable para la inclusión de mayor cantidad de mujeres al interior de los partidos y de la administración pública. De esa manera, la inclusión de las mujeres, tarde o temprano, se convierte en un impulso importante para seguir reduciendo la brecha y promoviendo la igualdad entre los géneros (RODRÍGUEZ, 2003).

$\mathrm{Si}$ existe entonces una expectativa del efecto positivo de una mayor representación femenina, este se debe ver reflejado en los indicadores más comunes de desigualdad de género. Para analizar ese efecto y realizar una comparación entre los países de los tres grupos vamos a basarnos en el Gender Empowerement Index (GEM), calculado por la Organización de las Naciones Unidas (ONU) con base en mediciones del ingreso relativo de las mujeres, su participación en las posiciones de alto nivel e influencia en el ámbito económico, así como su acceso a los cargos gubernamentales $^{2}$ (ONU, 2016).

${ }^{2}$ Cabe señalar que en 2010 el GEM fue sustituido por el Gender Inequality Index, un indicador compuesto y más complejo que el previo, que incluye las mediciones del acceso a la salud reproductiva, empoderamiento y participación en el mercado laboral. 
Grafico 2 - Las desigualdades de género

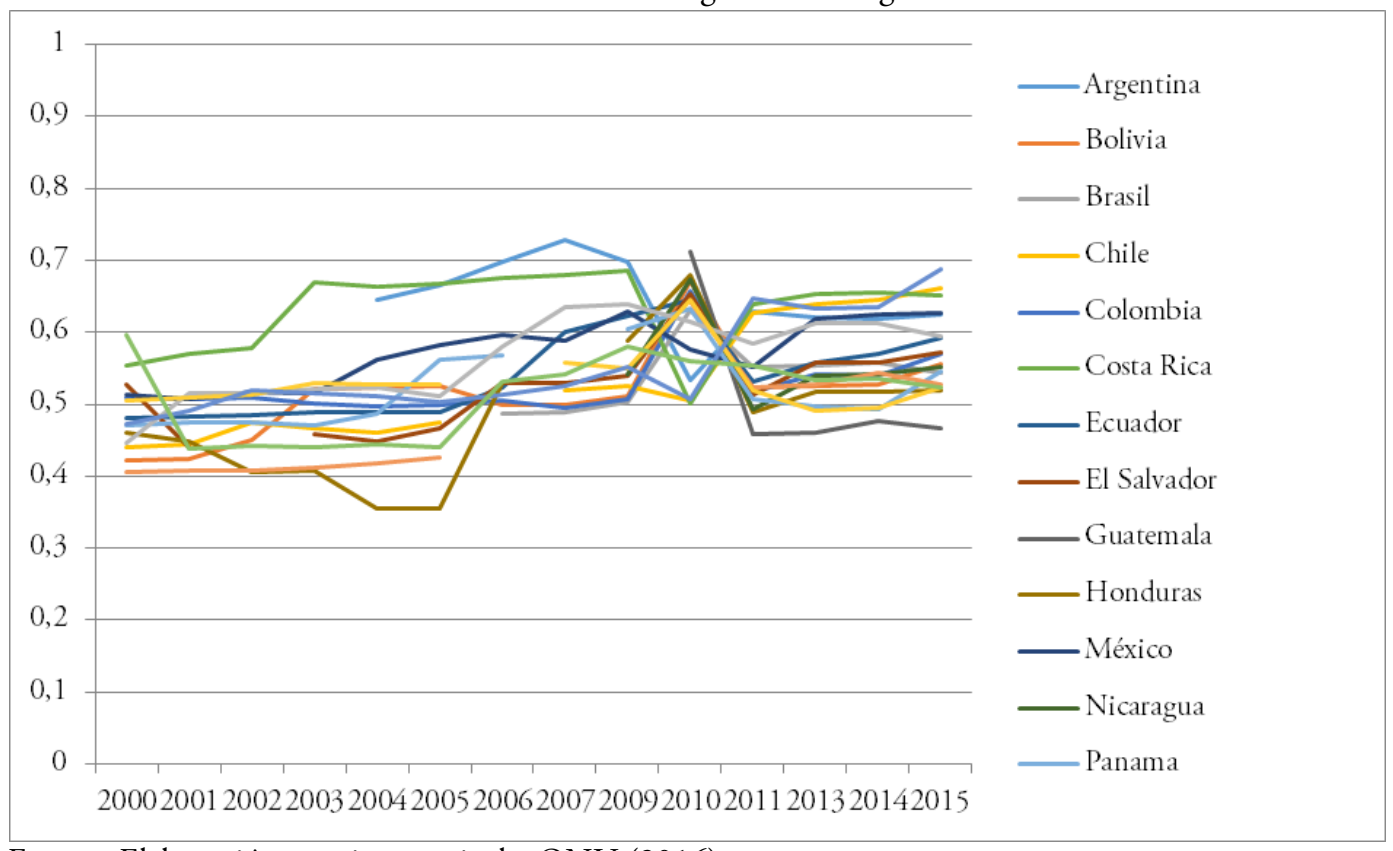

Fuente: Elaboración propia a partir de: ONU (2016).

Los datos señalan que la región es relativamente homogénea en cuanto a los niveles de desigualdad de género y que, en los últimos 15 años, no se produjo un mayor avance, al pasar el promedio regional de 0.486 a 0.574 (mientras el valor este más cercano a la unidad, existe menor desigualdad), mientras que la representación de las mujeres se duplicó, pasando desde un 12 al 26\%.

Analicemos entonces nuestros casos, comparando los niveles de la representación femenina con los de desigualdad de género: 
Tabla 1 - Cuotas, representación y desigualdad

\begin{tabular}{|c|c|c|c|}
\hline Efecto de la cuota & País & Representación & Desigualdad \\
\hline \multirow{5}{*}{$\begin{array}{l}\text { Fuerte } \\
>40 \%\end{array}$} & Bolivia & $53.10 \%$ & 0.556 \\
\hline & México & $42.40 \%$ & 0.627 \\
\hline & Ecuador & $41.60 \%$ & 0.593 \\
\hline & Nicaragua & $41.30 \%$ & 0.551 \\
\hline & Promedio & $44.60 \%$ & 0.582 \\
\hline \multirow{4}{*}{$\begin{array}{l}\text { Elevado } \\
30-39 \%\end{array}$} & Argentina & $36.60 \%$ & 0.624 \\
\hline & Costa Rica & $33.30 \%$ & 0.651 \\
\hline & El Salvador & $32.10 \%$ & 0.573 \\
\hline & Promedio & $34.00 \%$ & 0.616 \\
\hline \multirow{4}{*}{$\begin{array}{l}\text { Moderado } \\
20-29 \%\end{array}$} & Honduras & $25.80 \%$ & 0.520 \\
\hline & Perú & $22.30 \%$ & 0.594 \\
\hline & República Dominicana & $21.90 \%$ & 0.523 \\
\hline & Promedio & $23.33 \%$ & 0.546 \\
\hline \multirow{9}{*}{$\begin{array}{l}\text { Débil } \\
<20 \%\end{array}$} & Colombia & $19.90 \%$ & 0.571 \\
\hline & Panamá & $18.30 \%$ & 0.546 \\
\hline & Venezuela & $17.00 \%$ & 0.524 \\
\hline & Uruguay & $16.20 \%$ & 0.687 \\
\hline & Chile & $15.80 \%$ & 0.662 \\
\hline & Paraguay & $15.00 \%$ & 0.528 \\
\hline & Guatemala & $13.90 \%$ & 0.467 \\
\hline & Brasil & $9.90 \%$ & 0.543 \\
\hline & Promedio & $15.75 \%$ & 0.566 \\
\hline
\end{tabular}

Fuente: Elaboración propia a partir de IPU (2016) e ONU (2016).

Contra la predicción intuitiva, podemos observar que los países con mayores niveles de representación femenina no producen las políticas más igualitarias. Nicaragua y Bolivia, dos países con mayor representación, tienen niveles de desigualdad de género mayores que el promedio regional (que es de 0.570); al mismo tiempo, dos de los países con representación femenina por debajo del 20\% y, por ende, por debajo del promedio regional (26\%), Uruguay y Chile, son los dos países con mayor nivel de igualdad de género.

Así, nuestro análisis nos lleva a una conclusión reservada sobre el funcionamiento de las cuotas. Por un lado, podemos notar su impacto en elevar el 
número de mujeres en los cargos públicos, siempre y cuando la cuota sea diseñada de una manera que permita su aplicación efectiva. Por el otro, la expectativa de que una mayor presencia de las mujeres en los cargos de representación cambie la política (la manera de hacerla y sus resultados) no necesariamente es cierta.

Ahora bien, ¿por qué en la región que no se produjo el efecto esperado y la representación femenina no derivó en una mejora significativa de la igualdad entre géneros? Una primera respuesta seńala a que la mayoría de los estudios que demuestran una relación positiva entre ambos fenómenos se basan en los casos de Estados Unidos y algunos países europeos. Es posible que las estructuras del poder y la dinámica de la política en América Latina afecte el desenvolvimiento de las mujeres, afectando su capacidad de adoptar una agenda de género.

Por otro lado, otras investigaciones, como el trabajo de Camp sobre las elites políticas mexicanas, señalan que las mujeres de más altos niveles del ejercicio del poder tienen antecedentes, experiencias, desempeño y proyectos políticos indistinguibles de los hombres, a pesar de que muchas de ellas han participado en acciones a favor de la equidad de género. Su conclusión es que los hombres y mujeres se involucran en la política por las mismas razones: la excitación y el ejercicio del poder (CAMP, 1998). Por otro lado, la investigación de Rodríguez apunta a que las mujeres, en primer lugar, se apegan a la agenda del partido, dedicando sus mayores esfuerzos a lograr los objetivos comunes, por lo que el tema de género casi siempre viene en un segundo lugar, dando prioridad a los temas de la política "en general": pobreza, trabajo, derechos humanos, educación, política social, salud, etc.

Finalmente, puede ser que los casos de la región confirman un fenómeno descrito también en la literatura conforme al cual un incremento importante en la representación femenina puede generar un retroceso en las políticas públicas y que, contra intuitivamente, las mujeres pueden actuar con mayor eficacia cuando forman grupos pequeños. Esto se debe a que las mujeres pueden movilizarse tanto individualmente, como apoyarse en grupos de electores para lograr sus objetivos (CROWLEY, 2004; REINGOLD, 2000; THOMAS, 1991). Esas opciones se desdibujan al incrementarse el número de legisladoras femeninas, quienes se ven obligadas a modificar las estrategias y a enfrentarse a los líderes masculinos. Sin embargo, no necesariamente cuentan con experiencia y capacidades requeridas para ello, evidenciando que, aunque las cuotas dan presencia a las mujeres en términos numéricos, por si solas no garantizan liderazgo ni influencia real en la política. La obligación de postular a las candidatas femeninas cambia las estrategias de los partidos y la dinámica de su vida interna, pero frecuentemente, en lugar de promover a las 
mujeres con experiencia política y cierto liderazgo, prefieren colocar en las listas a otros perfiles, que les parecen más manejables. En ese sentido, es importante hacerse cargo de las diferencias que existen entre las mujeres y reconocer que no todas ellas se suman a la agenda de género, ya que sus preferencias ideológicas o vínculos partidistas resultan determinantes para sus posturas políticas. El hecho de ser mujer no conlleva ni una experiencia de vida única, ni la misma preferencia por las políticas públicas.

Esas observaciones y la experiencia latinoamericana parecen respaldar a los críticos de la teoría de la "masa crítica". Childs y Krook, recopilando las aportaciones de esos autores, señalan que los números no tienen efectos mágicos y que el incremento en la representación política de las mujeres no tiene como un resultado natural y directo la construcción de una representación sustantiva y una democracia paritaria (CHILDS y KROOK, 2008). Parece que otros factores, como oportunidades y limitaciones de la afiliación partidista (CHILDS, 2004), membresía en los comités legislativos (NORTON, 1995), normas institucionales (ROSENTHAL, 1998), falta de experiencia legislativa (COWLEY y CHILDS, 2003), así como los factores externos, pueden resultar determinantes para el desempeńo de las mujeres legisladoras (CHILDS y KROOK, 2008).

\section{Conclusiones}

Esta breve mirada a la relación entre la representación femenina y una mayor igualdad de género en términos generales nos permite concluir que la relación esperada entre ambos no se presenta. Más mujeres en los parlamentos no necesariamente se traducen en políticas públicas con perspectiva de género. En términos más generales, parece ser que la ruta hacia la democracia paritaria no pasa por una igualdad numérica y conquista de los espacios del poder.

Esa conclusión, aunque requiere de futuras investigaciones para conocer a mayor profundidad si, en qué medida y de qué manera cambian los patrones de la política en los países con mayor representación femenina, nos traza una agenda a futuro. Ante el fallo de la premisa de los efectos de las cuotas, según la cual la inclusión de las mujeres, tarde o temprano, se convierte en un impulso importante para seguir reduciendo la brecha y promoviendo la igualdad entre los géneros, será necesario un cambio de estrategia. Los países que apostaron por la ruta políticoelectoral deberán modificar el enfoque y poner un mayor peso a los demás ámbitos de la vida social.

Al parecer, la construcción de una democracia paritaria no pasa por la ruta electoral. 
Karolina Monika Gilas é Doutora em Ciências Politicas e Sociais pela Universidade Nacional Autônoma do México. É investigadora do Tribunal Eleitoral do Poder Judiciário da Federação, do México. E-mail: karolina_gilas@hotmail.com.

\section{Referências}

AGACINSKI, Sylviane. Politica de los sexos. Madrid: Taurus, 1998.

APARICIO, Francisco Javier; LANGSTON, Joy. Evaluación de la perspectiva de género en plataformas de partidos politicos, candidaturas y cargos de elección 2009. Informe final. México, D.F.: CIDE, 2009.

BRATTON, Kathleen A. Critical Mass Theory Revisited: The Behavior and Success of Token Women in State Legislatures. Politics and Gender, v. 1, n. 1, p. 97-125, 2005.

CAMP, Roderic Ai. Women and Men, Men and Women: Gender Patterns. In: RODRÍGUEZ, Victoria E. Women's Participation in Mexican Political Life. Boulder: Westview Press, 1998. p. 167-178.

CERVA, Daniela; ANSOLABEHERE, Karina. Trabajo introductorio: protección de los derechos político-electorales de las mujeres. In: REZA, Enrique Ochoa. Género y derechos políticos. La protección jurisdiccional de los derechos político-electorales de las mujeres en México. México, D.F.: TEPJF, 2009.

CHILDS, Sarah. New Labour's Women MPs: Women Representing Women. New York: Routledge, 2004.

CHILDS, Sarah; KROOK, Mona Lena. Critical Mass Theory and Women's Political Representation. Political Studies, v. 56, p. 725-736, 2008.

COBO, Rosa. Multiculturalismo, democracia paritaria y participación política. Política y Sociedad, Madrid, n. 32, p. 53-65, 1999. Disponible en: <http://www.cdd.emakumeak.org/ficheros/0000/0518/POSO9999330053A.pdfs. Acceso en: 10 feb. 2016.

COWLEY, Paula; CHILDS, Sarah. Too Spineless to Rebel? New Labour's Women MPs. British Journal of Political Science, v. 33, n. 3, p. 345-365, 2003.

CROWLEY, Jocelyn Elise. When Tokens Matter. Legislative Studies Quarterly, v. 29, n. 1. p. 109-136, 2004.

DAHLERUP, Drude. From a Small to a Large Minority: Women in Scandinavian Politics. Scandinavian Political Studies, v. 11, n. 4, p. 275-97, 1998.

DAHLERUP, Drude. Women, Quotas and Politics. New York/London: Routledge, 2006. 
DAHLERUP, Drude; FREIDENVALL, Lenita. Quotas as a 'Fast Track' to Equal Representation of Women: Why Scandinavia is No Longer the Model. International Feminist Journal of Politics, v. 7, n. 1, p. 26-48, 2005.

DAHLERUP, Drude; FREIDENVALL, Lenita. Electoral Gender Quota Systems and Their Implementation in Europe. Brussels: European Parliament, 2008. Disponible en: <http://www.europarl.europa.eu/document/activities/cont/200903/20090310ATT51390/2009 0310ATT51390EN.pdf>. Acceso en: 7 feb. 2016.

DONANRD, Giselle. Se reappropier la politique par la parité. Partié-Infos, n. 8, dez. 1994.

FOX, Richard L.; SCHUHMANN, Robert A. Gender and Local Government: A Comparison of Women and Men City Managers. Public Administration Review, v. 59, n. 3, p. 231-242, 1999.

GONZÁLEZ, Manuel et al. Hacia una democracia paritaria: La evolución de la participación política de las mujeres en México y sus entidades federativas. México: TEPJF, 2016.

IDEA; US; IPU. Atlas of Electoral Gender Quotas. Geneva: IPU, 2013.

IPU. Women in Parliament Database. Inter-Parliamentary Union, 2016. Disponible en: <http://www.ipu.org/wmn-e/world.htm>. Acceso en: 7 feb. 2016.

KANTER, Rosabeth Moss. Some Effects of Proportions on Group Life. American Journal of Sociology, v. 82, n. 5, p. 965-990, 1977.

KATHLENE, Lyn. Alternative Views of Crime: Legislative Policymaking in Gendered Terms. Journal of Politics, v. 57, n. 3, p. 696-723, 1995.

KRENNERICH, Michael. ¡Mujeres al parlamento! Sistemas electorales y cuotas de género en la mirilla. In: NUNEEZ, Reynoso. La democracia en su contexto. México: UNAM, 2009. p. 173205.

LAWLESS, Jennifer L.; FOX, Richard L. Men Rule: The Continued Under-Representation of Women in U.S. Politics. Washington, DC: Women \& Politics Institute, 2012. Disponible en: <http://www.american.edu/spa/wpi/upload/2012-Men-Rule-Report-web.pdf>. Acceso en: 8 feb. 2016

LEUCHPRECHT, Peter. El acceso de las mujeres a los procesos de adopción de decisiones políticas. In: MARIÑO MENÉNDEZ, Fernando (Ed.). La protección internacional de los derechos de la mujer tras la Conferencia de Pekin. Madrid: Universidad Carlos III de Madrid, 1996. p. 123-128.

MEDINA TORRES, Luis Eduardo. Cuotas electorales de género e integración de congresos. México: UNAM-IIJ y TEPJF, 2011.

MOREIRA, Constanza; JOHNSTON, Niki. Democracia, género y equidad: Aportes para el debate sobre los mecanismos de acción afirmativa. Montevideo: Friedrich Ebert StiftungUruguay, 2003. Disponible en: <http://library.fes.de/pdf-files/bueros/uruguay/01819.pdf>. Acceso en: 17 mar. 2016

NORRIS, Pippa. Recruitment. In: KATZ, Richard; CROTTY, William (Eds.). Handbook of Party Politics. London: Sage, 2006. p. 89-108.

NORTON, Noelle. Women, It's Not Enough to be Elected: Committee Position Makes a Difference. In: DUERST-LAHTI, Georgia; KELLY, Rita Mae (Eds.). Gender Power, Leadership, and Governance. Ann Arbor MI: University of Michigan Press, 1995. p. 115-140. 
NORTON, Noelle. Uncovering the Dimensionality of Gender Voting in Congress. Legislative Studies Quarterly, v. 24, n. 1, p. 65-86, February 1999.

ONU. Human Development Reports 2000-2015. 2016. Disponible en: <http://hdr.undp.org/en/global-reports>. Acceso en: 11 feb. 2016.

PESCHARD, Jacqueline. El sistema de cuotas en América Latina. Panorama general. IDEA: Estocolmo, 2002.

REINGOLD, Beth. Representing Women. Chapel Hill NC: University of North Carolina Press, 2000.

REY MARTÍNEZ, Fernando. Cuotas 2.0. Un nuevo enfoque de las cuotas electorales de género. México: TEPJF, 2013.

RODRÍGUEZ, Victoria E. Women in Contemporary Mexican Politics. Austin: University of Texas Press, 2003.

ROSENTHAL, Cindy Simon. When Women Lead. New York: Oxford University Press, 1998.

SCOTT, Joan W. Parité! Equidad de género y la crisis del universalismo francés. México, D.F.: FCE, 2012.

THOMAS, Sue. The Impact of Women on State Legislative Policies. Journal of Politics, v. 53, n. 4, p. 958-976, 1991.

THOMAS, Sue. The Effects of Race and Gender on Constituency Service. Western Political Quarterly, v. 45, p. 161-180, 1992.

THOMAS, Sue. How Women Legislate. New York: Oxford University Press, 1994.

TOLLESON Rinehart, Sue. Do Women Leaders Make a Difference? Substance, Style and Perceptions. In: DODSON, Debra (Ed.). Gender and Policy Making. New Brunswick, NJ: Center for American Women and Politics, 1991.

UNIÓN EUROPEA. Declaración de Atenas. Atenas, 3 nov. 1992. Disponible en: <http://www.urv.cat/media/upload/arxius/igualtat/JeanMonnet/2013/Lectura_recomendada_D eclaracion_Atenas_1992.pdf> Acceso en: 17 mar. 2016

ZÚNIGA, Yanira. Democracia paritaria: de la teoría a la práctica. Revista de Derecho, v. 18, n. 2, p. 131-154, Diciembre de 2005. Disponible en: <http://www.scielo.cl/scielo.php?script=sci_arttext\&pid=S0718-09502005000200006>. Acceso em: 11 feb. 2016.

Texto recebido em 29 de fevereiro de 2016. Aprovado em 17 de março de 2016. 